\title{
Energy Efficient Routing Protocol By One Way Multi-Hope Sensor Nodes
}

\author{
${ }^{1}$ Administrative and Financial Directorate \\ Ministry of Higher Education and Scientific Research \\ Baghdad, Iraq \\ heba_alhayani@yahoo.com \\ ${ }^{2}$ Administrative and Financial Directorate \\ Ministry of Higher Education and Scientific Research \\ Baghdad, Iraq \\ boraqfade180@gmail.com \\ ${ }^{3}$ Scholarship and Cultural Relations Directorate \\ Ministry of Higher Education and Scientific Research \\ Baghdad, Iraq \\ marwah.k.farhan@gmail .com
}

${ }^{1}$ Heba Hussain Hadi, ${ }^{2}$ Buraq Fadhil Neamah, ${ }^{3}$ Marwa Khaleel Farhan

Article History: Received: 11 January 2021; Revised: 12 February 2021; Accepted: 27 March 2021; Published online: 16 April 2021

\begin{abstract}
Multi-hope is widely used for data aggregation and transmission in various applications. The resource availability determines the life time of a WSN. Sensor nodes are powered by a tiny battery that supplies the required energy for the sensor and transmitter. The residual energy available at any moment decides the fitness of the sensor node. The sensor node senses the environment and transmits the data to the sink. Efficient data transmission and aggregation with less energy consumption can prolong the lifetime of the sensor network. The sensor node that is inside the coverage area of the sink can directly transmit the data to sink in a single-hop transmission. The sensor node that is not inside the coverage area should transmit the data to the neighbor node which is in the coverage area of the sink. The data is then turn transmitted by the node close to it fall in multi-hop transmission involving a number of intermediate nodes to forward the data to the sink and consumes extra energy for the forwarding process. The formation clusters and data transmission of data by Cluster Heads $(\mathrm{CH}) \mathrm{can}$ eliminate many nodes involved in the transmission of same data. Clusters are a group of self-organized nodes in a geographic location that can communicate among them. A node in a cluster with higher residual energy will be acting as $\mathrm{CH}$ and all other nodes in the cluster transmit the data to the $\mathrm{CH}$. The $\mathrm{CH}$ transmits the aggregated data to the sink. The $\mathrm{CH}$ transmits the data to the sink either in single-hop transmission or multi-hop transmission. The cluster head consumes more energy than other nodes in the cluster as it is involved in aggregation and transmission process.
\end{abstract}

Keywords: - Routing, Bit Error Rate, Image Transmission, Multi-hope sensor nodes, Image processing.

\section{Introduction}

The data in the ledger book have been migrated to magnetic storage devices after the invention of computers. A computer is used to create, store and edit data. Once the computers were interconnected, sharing of data was made possible. These interconnected computers are called computer networks in which a computer can send information to a particular computer in the network or broadcast to all computers in the network. This revolution ensures the availability of data anywhere and anytime. Data Communication is the terminology that out routed the delay in receiving information from any part of the world. A network may be considered as a set of devices connected through a link (medium). A node is a device which is capable of transmitting and receiving data generated by other nodes connected in the network. The links that connects the nodes are called communication channels.

The interconnection of computer forms a computer network. The data originated from one computer may reach any other computer in any part of the world that is connected in the network. Wired communication channels are very efficient and have restrictions on the establishment cost and physical barriers have the impact on the network. Wired network doesn't have the advantage of mobility and also the Quality of Service (QoS) offered is not as good as that of the wired networks. But the wireless networks are preferred to wire networks because of its easy establishment. The wireless networks demands only a Base Station (BS) establishment with an antennae and it can cover a radius up to few kilometers. It eliminates the laying of wires to connect the nodes. The wireless nodes within the coverage range of the BS can communicate and transmit the data among the nodes in that BS. If BSs are connected, a node in one BS can communicate with another node in a different BS. The BS connects with each node with different frequency channels. The energy consumed by the wireless networks is higher than that 
of the wired network. The major energy utilization is for the transmission and reception of the signals by the transceiver unit. The data loss is also higher when compared to the wired network. The easy establishment and the scaling factors have made the wireless network inevitable and find its application in all the areas where a communication is needed.

A fundamental set of WSNs, the Visual Sensor Networks (VSNs) are incorporated as camera detection gadgets, and consequently managing VSN recording and transmission of pictures. In our paper, the restriction degree to picture transmission in a remotely sensor networks [5]. The captured photo contains a highly dimensioned information to be transmitted that exceeds the sensor hub properties such as viscosity, suppressor factor, and temperature. When contrasted with other WSN applications, the process of broadcasting of the image in the sensor network destroysmore of the hub's battery. Along these lines heavy information traffic for remote picture transmission over sensor networks is reducing network lifetime [6]. To overcome such issues, picture pressure techniques aimed at improving network lifespan. The picture pressure for the sensor network is called the pressure detection (CS) process. WSNsas a couple of late process for photo transmissions believe for pressure detection and picture pressure. The greater part of the strategies depends on the understanding strategy called waveform change based picture pressure for the agreeable and non-supporting transmissions of picture [7]. Non-ancillary network-based picture pressure strategy does not affect the presentation of correspondence boundaries, such as the quality picture reproduction and the energyof productivity borders. In writing, the DWT technique is employed on several quality layers and strategies that inherently identify transmission and coding limitations. With estimates that remote hubs are single-iteration wire assets, auxiliary image transmission is widely used these days to meet energy reserve funds, with measurement and cost being mandatory. Be that as it may, the agreed image transmission bit error rate (BER) indicates significant effects of picture quality factors such as performance limitations, for example, MSE, zenith to signal the ratio noiseand so forth [8].

In agreeable correspondence, hand-off hubs go about as middle of the road hubs to send data from the source to the sink hub with fuses of information sending from source to objective. There are diverse helpful conventions planned like SDF (particular interpret and forward) just as IDF (steady unravel and forward). The SDF technique broadly embraced in agreeable interchanges in which hand-off hub is liable for accurately unravel and afterward just forward the messages. In IDF strategy the hand-off hub advances information to the objective hub on which objective hub required it. The energy decrease in agreeable interchanges made by limiting the exchange energy, yet extra RF coordinated circuit use across transmission hubs required [9]. The new examinations guarantee that the agreeable techniques are impressively better energy effectiveness when contrasted with the single jump, multi-bounce and brief distance based transmission plans [10].

To date, many of the ancillary correspondence-based illustrations are planned and evaluated by various, yet, exploratory holes are still evident especially when managing picture transmission over WSNs; probably for the reason that pictures may encompass fundamental data [11 ] [12]. This research explores the upgrading of the picture quality limits in multi-trust picture transmission agreed upon in WSNs. The creativity in the form of shared picture transformations capable of completing effective picture transmission and remaking will be presented. The curiosity of this work can be expressed as follows: First, we announced the picture in association to upgrade the standard of the picture during the broadcast on WSN. Image quality improvement also limits energy use. In addition, we adopted and planned discrete wavelet transform-2 dimensional (2D-DWT) for improved quality of the image. The previously referred effort 2D-DWT based and our work differ in a way that we have only approximated the 2DDWT strategybecause it holds the important image data in preparing other remote correspondence, for example, adjustment demodulation. Accordingly, the proposed computation helps to limit the additional computation and preparation power at the source, transfer, and objective centers. In the hands-off, we used the SDF method. Section II deals with the brief discussion on previous works represented. Research methodology is discussed briefly in section III followed by section IV where results and discussion are demonstrated. Eventually, the conclusion and recommended future work are presented in Section V.

\section{Related work}

This section presents the previous studies of methods of image transmission in the sensor networks.

The transmission technique picture [1], with thought of energy proficiency for WSNs is proposed. The planned two distinct methodologies included open-circle picture change procedure and shut circle. Those strategies set up upon wavelet picture change just as the semi-solid change for achieve power conservation. Wavelet picture change supplies data disintegration in numerous means of assurance. Henceforth picture can be isolated into bundles while sending in agreeable correspondences.

In [2], authors presented a novel picture pixel-area information based office apportioning procedure in grouping to improve picture change standard joined by supreme force recompense limitation for picture applications (petition) in WMSNs (Wireless Multimedia Sensor Networks). Organization offices obviously designated amid PHY, MAC, just as layers in APP about introduction fragment territory with capacity of power that is sure if picture change standard is improved. Reproduction results of this article have guaranteed the helpfulness of introduced way to deal with achieving incredible picture quality just as force capability. 
In [3], the helpful change technique for picture sensors in succession utilization intra-sensor associations for decide to change just as security circulating models set up on way variety proposed. The protected picture transmission and the force capability was the striking commitment created by the author. That technique didn't allow each picture sensor to move ideal parts of agreed pictures by means of pertinent change tracks in a force capable way, anyway additionally supplies different safeguarding to agreed picture regions through track inclination just as accommodative piece blunder rate (BER) imperative.

In [4], another technique for picture change in WSNs was introduced by means of acquiring the advantage of JPEG2000, be that as it may, picture shortening standard just as using MATLAB with C from Jasper through authors.

In [5], scientist addresses the strategy for naming helpful sign improvement to achieve power capable picture change in WSNs. A helpful sign improvement recommendation was persuading a direct result of its capacity to hold separate force use through broadening whole change use across different sensors. Autonomous bundles clarifying a submerged wavelet-scrambled picture present an exceptionally disparate gift toward picture standard. Through affecting this reality, they detailed strategy of likewise picking the no. of agreeable sensors for each parcel change in arrangement to get astounding possible picture standard expressed a restricted change power usage stipend.

In [6], an energy-mindful interleaving calculation was acquainted by authors with oversee part decrease response by broadening parcels as expressed each picture region's pre-figured change turnover. Creative results uncover that their strategy cannot simply create start to finish picture change procedure.

In [7], He proposed a power-capable picture-changing method for WSNs that fuses wave-based image disruption as a cooperative transmission. With this method the authors use a mixture of SDF (particularly decisive and so forth), so the transmission hub collaborates through converting only a minor-target class of the actual picture by connecting it with the source, obtained by DWT Has been done, that is, discrete wave transforms. The author declares that the additional force introduced the SDFDWT system is capable of multi-buoyancy as compared to non-collective single-jump, in addition to that which surpasses normal SDF processes. In addition, he announced the SDF-DWT strategy that it is efficient for energy when contrasted with customary multi-jump and single bounce. Their approach defeats current SDF technology issues. The author stated the need to save energy with the IDF (Incremental DF) strategy.

In [8], authors presented amazing Viterbi set up TVSD (complete variety succession discovery) to more noteworthy video/picture unscrambling in WSNs. Their recommended procedure uses the limited variety (BV) characteristic of pictures thus ideally obliged for reorganization. At last, it uncovered that organization set up Viterbi decoder can be selected for strong picture transformation using adjusted whole differentiation state just as division measurements.

In [9], authors presented a versatile FEC coding and joint effort flows transmission approach, the energy productivity and transmission amount is capacity to performing under the uncertain portable correspondence channel climate. These procedures address that the four period of their methodology. The near investigation shown that, proposed strategy proficiency for communicating better pictures by means of energy-limited cell phone conditions.

In [10], the force capable picture change point of view in remote media sensor networks planned. In the item, picture change across multi-bounce WSN was resolved to be conceivable, using a mix of force capable getting sorted out structure with a valid application layer convention which diminishes parcel blunder rate and retransmissions.

In [11], an inventive article vision imitation, just as picture change technique, was introduced for WMSN proposed. This methodology used change of picture part further whole picture. The recommended approach was assessed set up on in-hub power use just as changed PSNR. Re-enactment results of this technique guaranteed that the enhanced model devour the $95 \%$ of hub energy in the organization of acknowledged picture PSNR of 46 $\mathrm{dB}$ as separates with the other spot of craftsmanship takes after.

In [12] author depicted the mysterious key utilized in the encryption interaction is reliant on both the information and the boundary used to create the tumultuous guide. Subsequently the proposed plan can oppose picked plaintext assault. The critical space of the proposed plot is adequately enormous to defeat factual assaults. Exploratory outcomes and the security examination confirm the security and adequacy of the proposed pressure encryption conspire.

In [13] author Shows that experimental results have been given that the proposed technique can withstand the sine preparer attack, for example, JPEG, Gaussian, salt and pepper, histogram adjustment and hence similar presentation on the proposed procedure Is evaluated using. Benchmark programming checkmarks and methods are vigorously searched against checkmark attacks, for example, collages, trimmed means, hard and soft thresholds, wavelet compression, midpoint, projective, and wrap, etc.

In [14]-[17], authors talked about the various methodologies for information dependability, transmission, pressure for correspondence in remote sensor networks. 
In [18]-20], The techniques talked about in this segment shows their viability in an unexpected way; in any case, there are as yet couple of traps which need to address while managing great picture transmission agreeably in WSNs like inordinate energy utilization while handling proceeds with picture transmissions, to accomplish the compromise between picture quality and power of picture transmission. The greater part of the strategy zeroed in on fundamentally energy proficient picture transmission and secure picture transmission.

\section{Research Methodology}

The proposed model shows a single-direction multi-jump assisted picture transmission framework for remote organization. The transmitter (T) first improves picture quality, and the 2D-DWT method is used to pressure the picture. Ultimately, tweak and IFFT activities performed on the pack estimation class of the picture. Photo bundle sent for transfer over AWGN remote channel. The DF (decode-forward) process is applied on transfer hubs (RNs). In the collector hub (D), all cycles are switched to reproduce the first picture transmitted over a remote organization. For this model, the picture is sent in different sections via an auxiliary transfer hub. To effectively move the picture through the organization, each hand-off hub in the organization has two functions to perform a relay roll (RR) and a monitoring roll (MR). Each relay hub must have two limits. In Count 1, the information picture is either received on the transmitter or the collector is received from the transmitter to communicate at the remote organization. The goal of Count 1 is to improve the nature of the image which may be corrupted either by the capture of the gadget or in the remote transmission channel due to various enlightening conditions. To improve the quality of picture matter innovation to improve quality in this calculation, we used two picture transfer procedures, for example, Laplacian and general ablation. Figure 1 shows the resultant BER as well as PSNR and MSE measurement for the picture under examination.

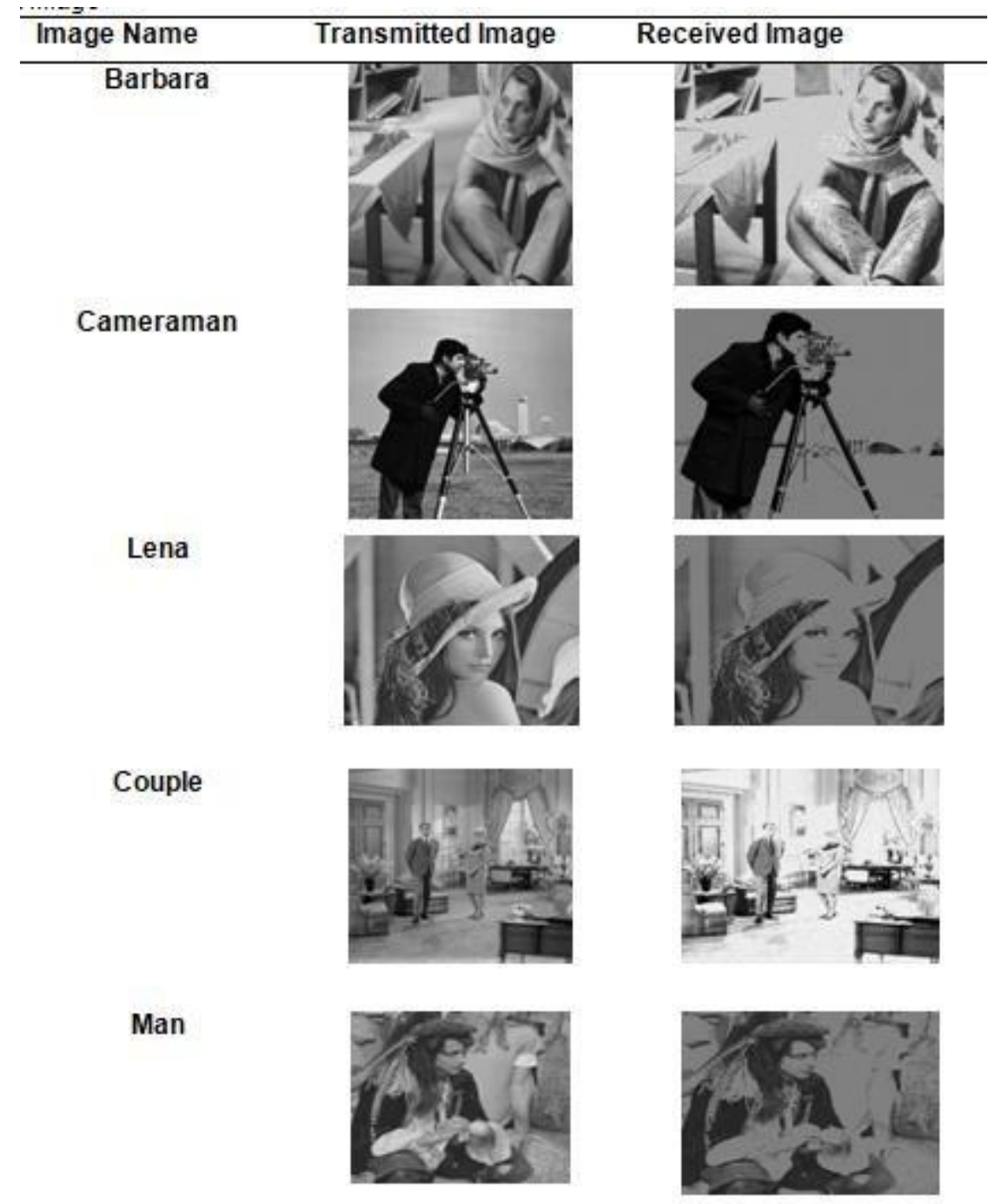

Fig.1. Reconstruction of Image 


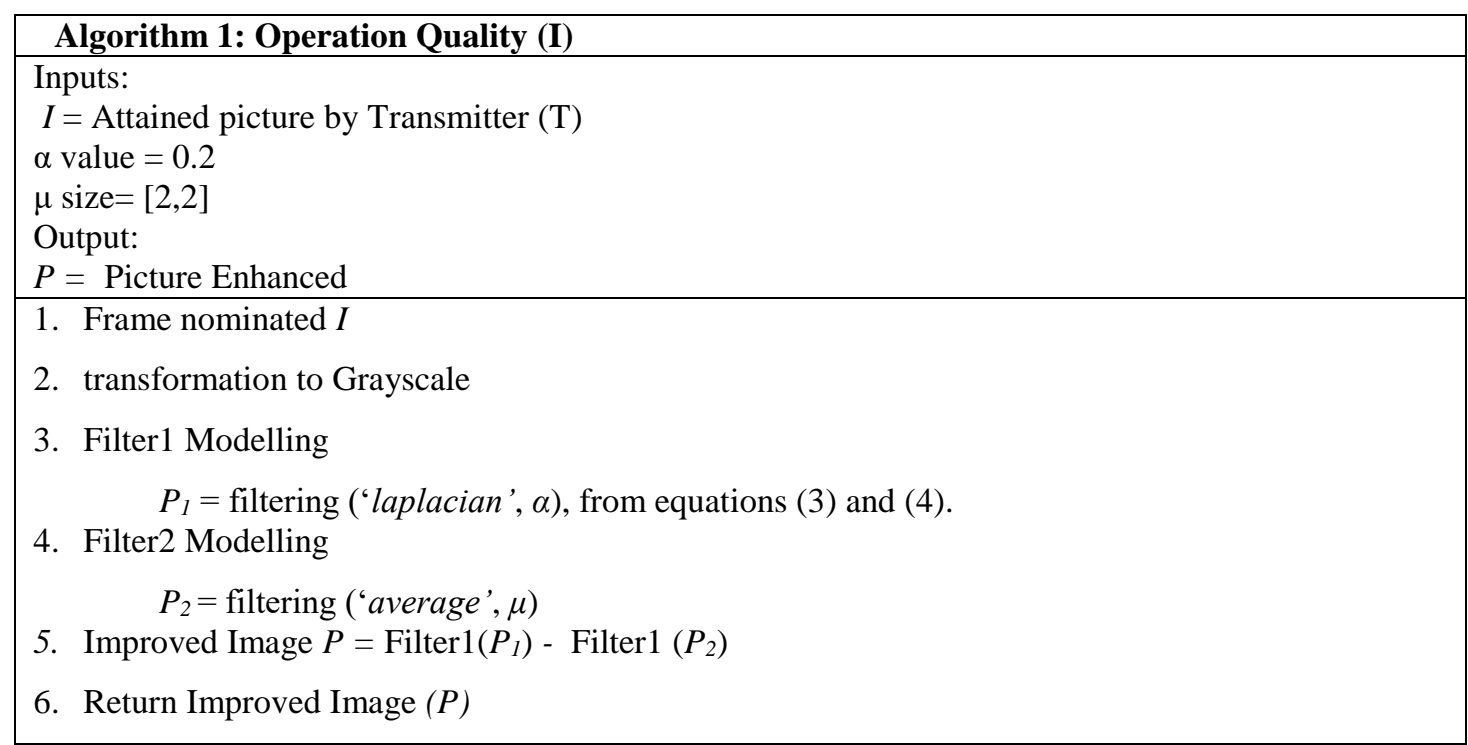

The Laplacian image on the input picture displays significant power change blanks. This Laplacian technique is used in habitual smoothing functions and all together affects the uproar. This capability takes a grayscale (2-D) picture as information and produces a separate grayscale (2-D) picture as a byproduct. Laplacian LF (p, q) of a pixel force esteems me photograph $(\mathrm{p}, \mathrm{q})$. Next, after Laplacian transfer, we implemented a general channel on the information diagram. This strategy is called mean separation. The normal channel is a direct spatial channel dependent on the sliding-window that gives the option of a median esteem in the window of an accurate estimate of the pixels in that assigned window. Results of the two channels then truncated in order obtain the ultimate pre-prepared image. The Algorithm 2 multi-bounce auxiliary diagram shows the transmitter end scheme in transmission measurements. Next, we need to perform the picture pressure, after pre-handling, showing Algorithm 2 where the 2D-DWT strategy was employed and addressed in the passage below. We chose the 2DDWT more than 1D- DWT in this effort. The advantages of employing 2D-DWT are revealed below:

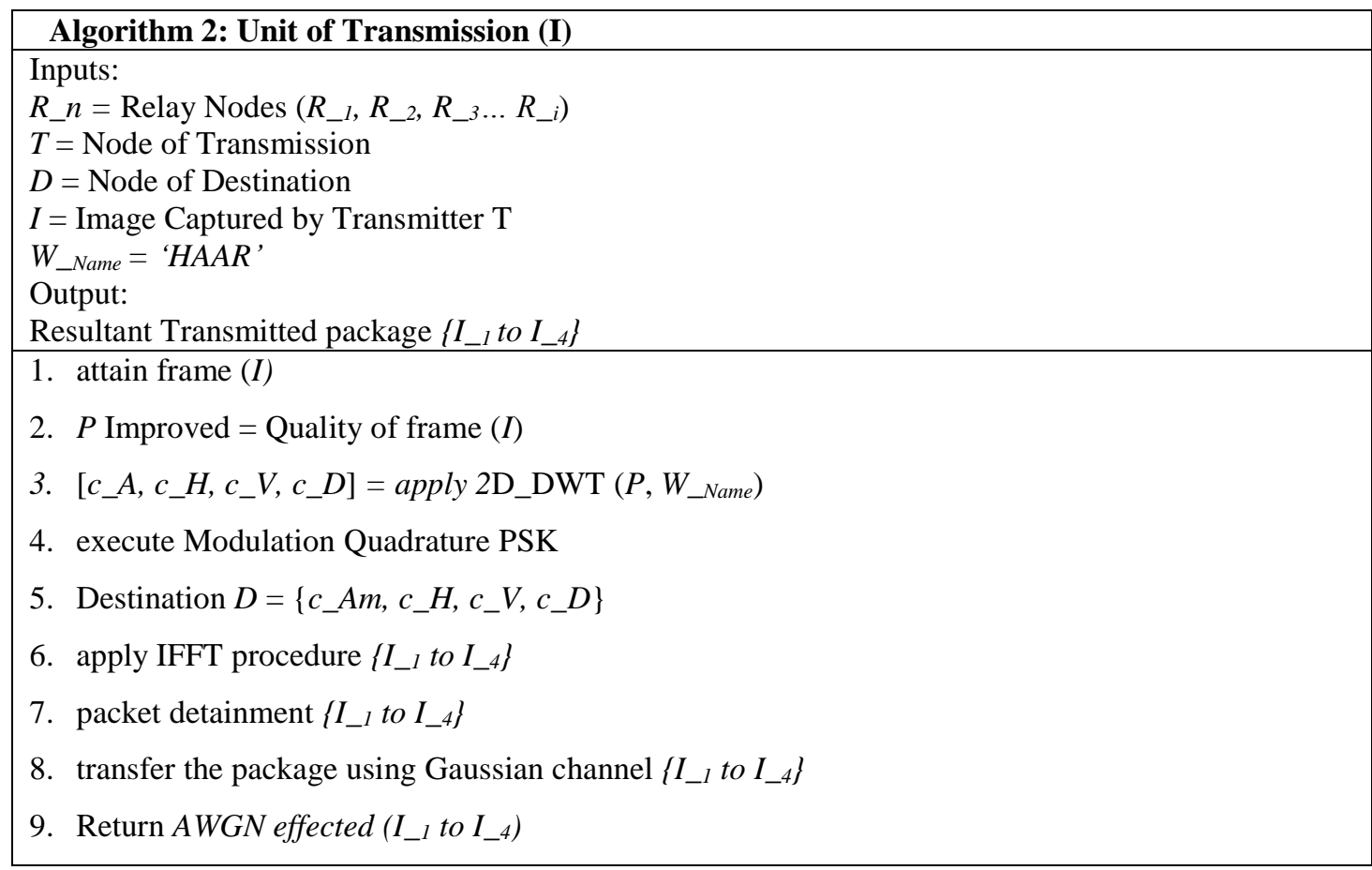

In one dimensional-DWT, the source picture of $\mathrm{N}$ sized is divided into two parameters, such as the parameter CA1 and the expansion parameter CD1. This can be accomplished using convolution operation using filters of low pass for lotion and high pass for expansion. Dyadic decommission is later used. The calculated distance of the parameter is:

$$
[0.5 *(N-1)]+L
$$


with $\mathrm{N}$ refers to how long and $\mathrm{L}$ to how wide the image is, respectively. As 1D-DWT is a single-level pressure process, this picture information is helpless against misfortune, and therefore 2-D DWT is used. In the multi-jump remotely transmitted image framework, we require effective pressured information techniques with minimum fail probabilities. In this way, at the transmitter side, we compressed the pre-handled picture using2D-DWT that is being employed widely in image preparation applications. This technique sports the rotations of the estimation coefficient at depth $\mathrm{j}_{\mathrm{j}}$ in quad elements: the estimation at the depth $\mathrm{j}_{+1}$, and referring to flat, vertical, and tilt methods. This pressure made about quad sections of information picture. In the last part of the beneficiary, the decomposition activity performed by the backward 2D-DWT. The reverse 2D-DWT, as the name suggests, reproduces the first image obtained from four information coefficients. Next to the pressure of the picture, next, we have to play the QPSK regulation following IFFT activity on the transmitter hub. QPSK is the phase conduction model and is used on the transmitter edge. The sum of the radio repetition ranges to convey the precision of QPSK is a large fraction of the binary phase shift keying signals. This justifies the additional subscribers on the channel. Thus this study will explore the QPSK tweak process. At the collector end, to obtain picture information using the QPSK demodulation strategy, demodulation is presented. Algorithm 3 works on each transfer hub which relies on the versatile DF technology. Each hand-off hub moves as either an RR hub or MR hub during picture transmission measurement. The square space is demonstrated by the DF technique using positions (7) and (8). The DF technique can provide the location capability as follows:

$$
f_{-D F}\left(O_{S R}\right)=r^{\wedge}
$$

$\mathrm{r}^{\wedge}$ refers to the identified or decoded information at the transfer hub, OSR addresses the first information from $S$ to recipient $\mathrm{R}$ for work $\mathrm{f} \_\mathrm{DF}$. Regarding QPSK regulation DF location capacity can be assumed as:

$$
f_{-D F}\left(O_{-} S_{R}\right)=\operatorname{sgnl}\left(O_{S R}\right)
$$

sgn (I) is the signal strength which displays QPSK ruling. Location work relied on OSR information, and there was no data about the nature of the S-R channel. Assuming that classes are effectively distinguished on each transfer, FFT is estimated on all DWT blocks with the exception of blocks. On the inference block, demodulation was performed. The process continued with the application on wavelet on the estimation class to reduce clutter from image information (which perhaps appropriate to different organization conditions). High-repetition reflects wavelet with decreasing commotion. This cycle applied to estimate the coefficients because it contains significant data compared to other coefficients of DWT. The means of denoting wavelet are: Where, $\alpha$ is an incentive to tarnish the base edge, the excess of the $0<$ of $<1$ is the change factor and the $I_{-} 1^{\wedge} \mathrm{R}(\mathrm{i}, \mathrm{j})$ region $(\mathrm{I}, \mathrm{J})$. In the event that the identities of the squares have faded, the current squares are disposed to describe the picture information

\begin{tabular}{|c|c|}
\hline \multicolumn{2}{|c|}{ Algorithm 3: Unit of Relay $\left(I \_1, I \_2, I \_3, I \_4\right)$} \\
\hline \multicolumn{2}{|c|}{$\begin{array}{l}\text { Inputs: } \\
R \_n=\text { Relay Nodes }\left(R \_l, R \_2, R \_3 \ldots R \_i\right) \\
I \_n=\text { Received Blocks }\left(I \_l, I \_2, I \_3 \ldots I \_i\right) \\
\text { Output: } \\
\text { Transmitted package }\left\{I \_l \text { to } I \_4\right\}\end{array}$} \\
\hline 1. & FOR each Relay $R \_n$ \\
\hline 2. & Discover received package using equations (6) and (7). \\
\hline 3. & IF discover is true \\
\hline 4. & Package $\left\{I \_l\right.$ to $\left.I \_4\right\}=$ apply $\left(\mathrm{fft}\left(I \_n\{2,3,4\}\right)\right)$ \\
\hline 5. & $Q P S K$ Demodulation $I_{1}=(\operatorname{In}\{1\})$ \\
\hline 6. & perform de-noising wavelet for $\left(I_{-l}\right)$ Equation (9) \\
\hline 7. & ELSE \\
\hline 8. & $\operatorname{drop}\left(I \_l\right)$ \\
\hline 9. & break \\
\hline 10. & END IF \\
\hline 11. & Perform modulation $\left(I_{-l}, Q P S K\right)$ \\
\hline 12. & $\mathrm{~F}_{\mathrm{r}}=$ Transmitted $\left\{I_{-1}, I_{-2}, I_{-3}, I_{-4}\right\}$ \\
\hline 13. & Execute through AWGN effect $\left\{F_{-} r\right\}$ \\
\hline 14. & END FOR \\
\hline
\end{tabular}
as unfortunate. In progress, performed re-balancing and IFFT tasks and communicated to the next surge. 
The interaction is rehashed among every one of the transfers still the expected beneficiary gets all the picture blocks. The cycle at beneficiary hub clarified in Algorithm 4 beneath. Recipient side, achieve backwards DWT to reproduced the picture from the got blocks. To stifle the clamor embedded in remote information move stage, the application of the algorithm 1 is employed once again so as to enhance the quality of the got picture.

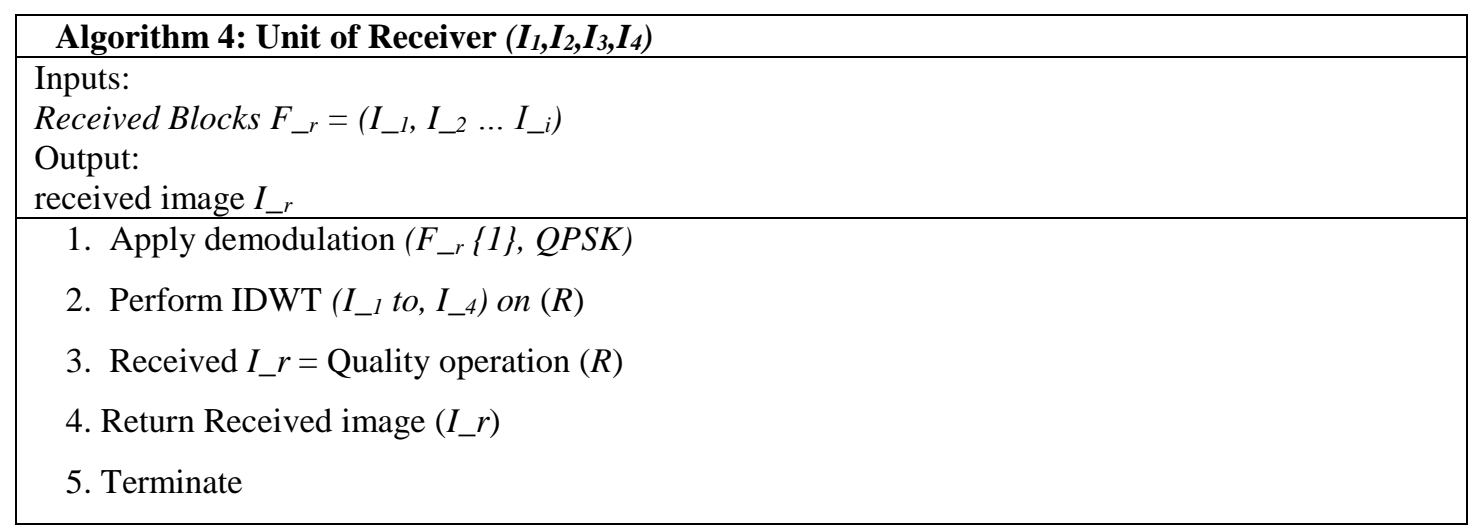

\section{Results and discussion}

We have led the arrangement of trial investigation utilizing the recommended approach for employing MATLAB with both 1D and 2D DWT. For this we tried and valued different diagrams to measure key performance of PSNR, MSE and BER. Results suggest that the developed strategy has improved all the status of craftsmanship techniques introduced in the ongoing past. In the event that PSNR results demonstrated some improvements, with MSE and BER are similarly better for the proposed technique as looked at condition of-workmanship picture transmission strategies. The end result of PSNR has shown improvement whilst utilizing the proposed technique probably as the effect of the utilization of wavelet De-noising just as discrete commotion sifting capacities at transmitter and beneficiary. Beneath figures are showing the diagrams for various reproduction setups. The outcomes are reflected in Fig.1.

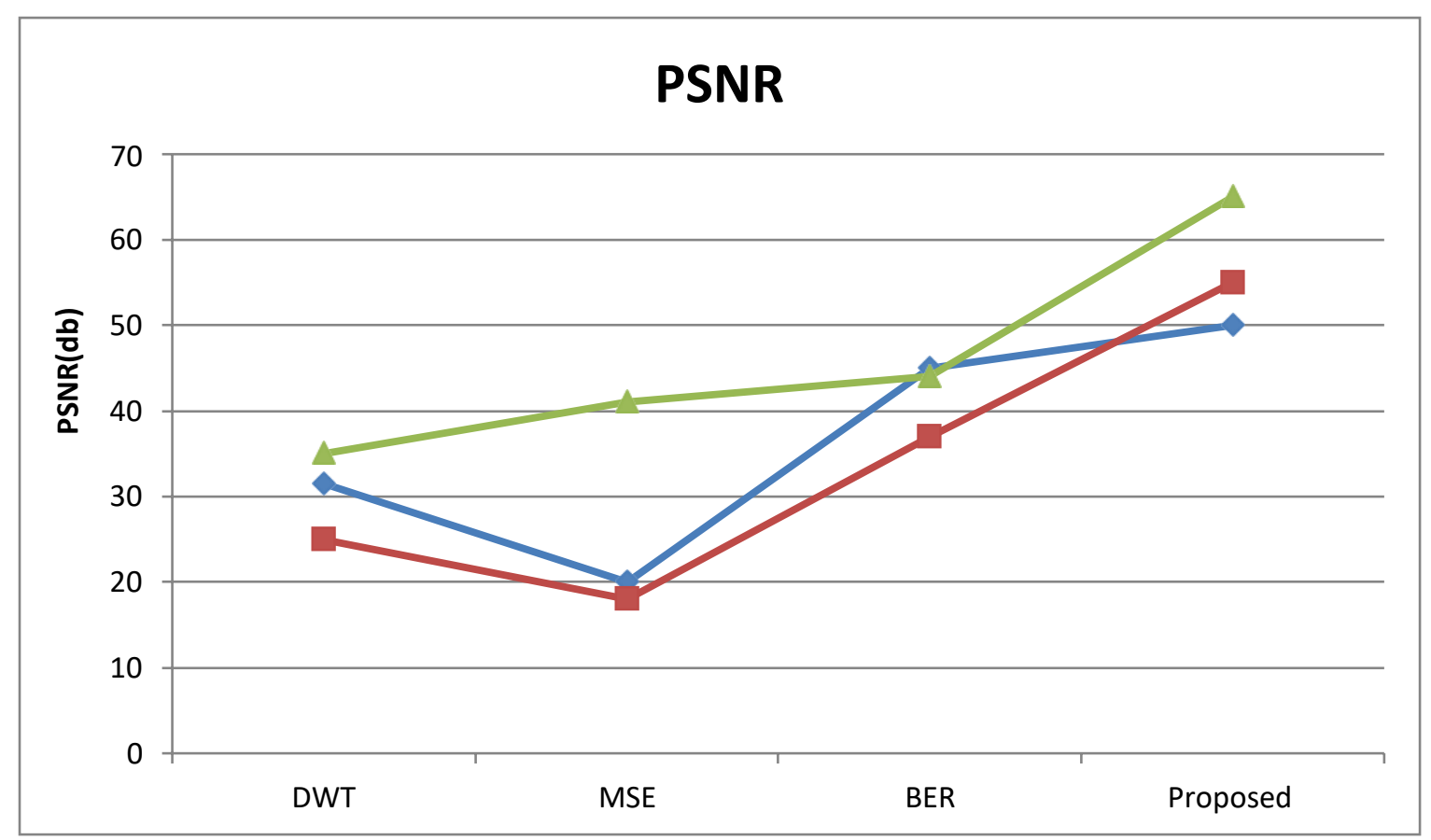

Figure 1: Comparative outputs of PSNR 


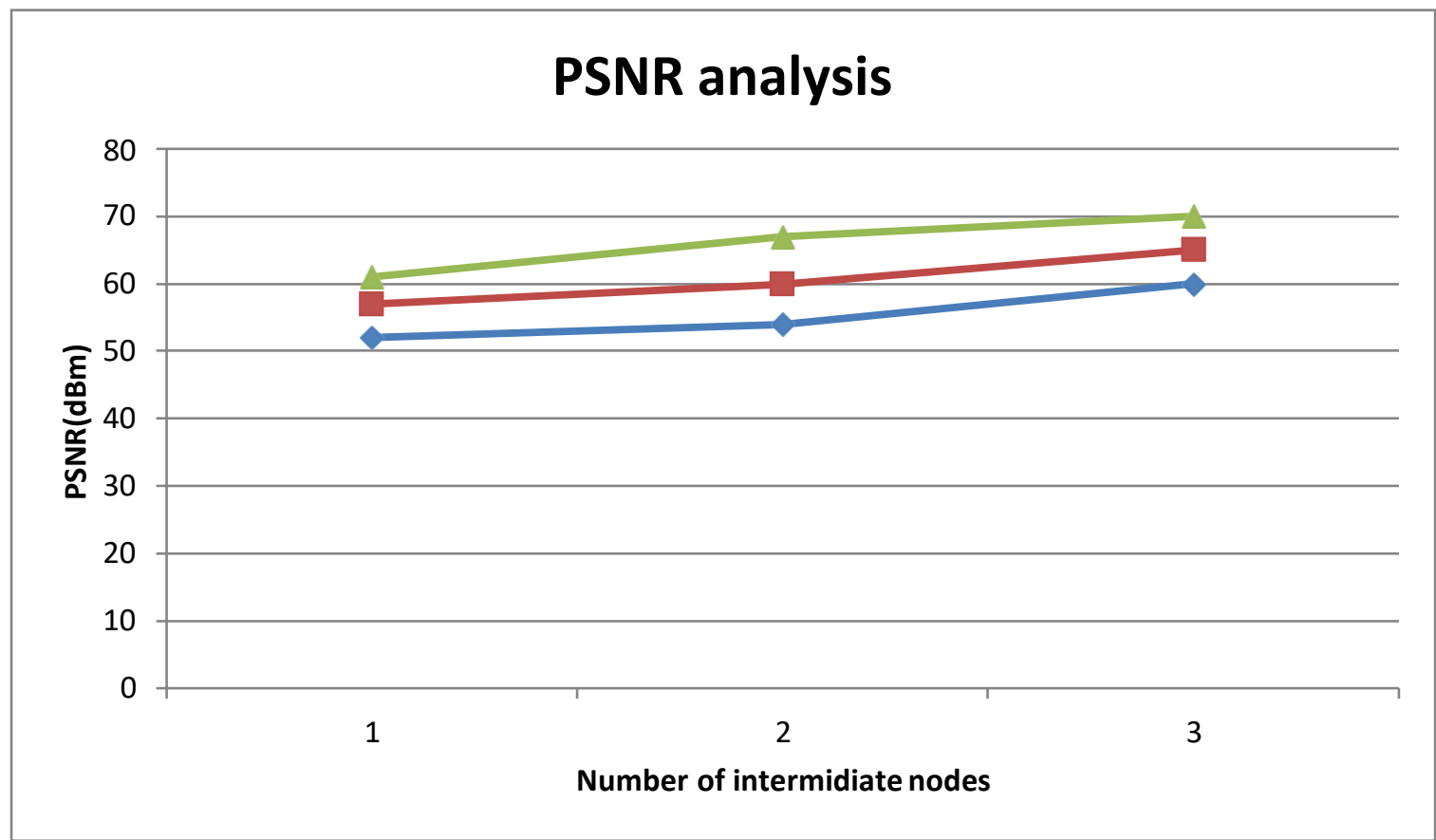

Figure 2: intermediate nodes vs. PSNR

Figure 2 and 3 depict the results of PSNR and MSE at each transfer hub which is expanding from 1 to 25 . In the event that the quantity of intermediates hubs rising, the picture nature of communicating is diminishing. Our proposed strategy showing the better exhibition in the two charts, as it was normal PSNR ought to be more and MSE is ought to be least.

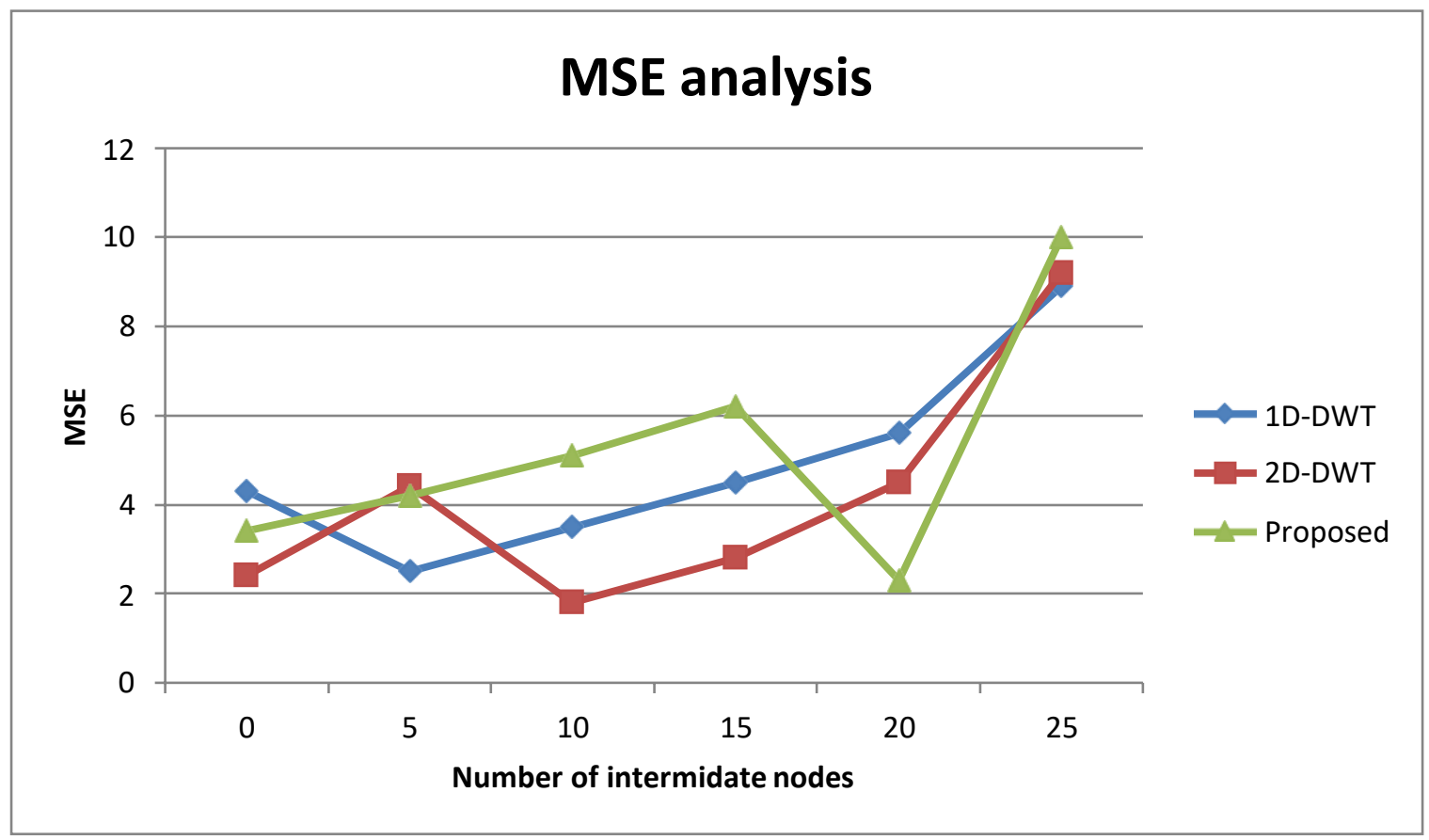

Figure 3: the relationship between intermediate nodes and MSE

Additionally, BER consequences of the suggested framework reveal that at every SNR level, the presentation of BER is fewer when contrasted with condition of workmanship arrangements appearing in figure 4. 


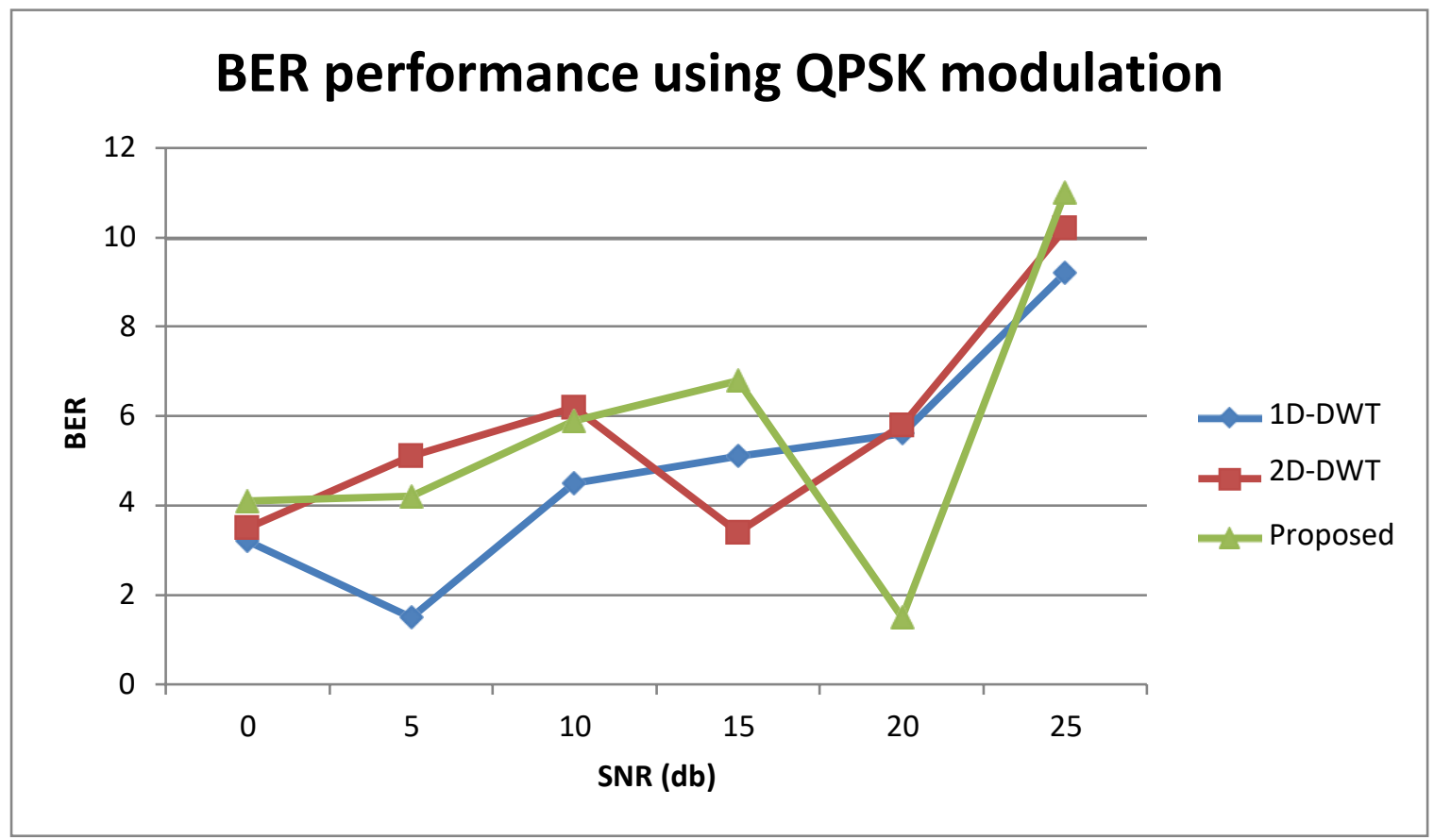

Figure 4: performance analysis of SNR vs. BER

\section{Conclusion and Future Work}

The movement of the sink paved the way for an effective data aggregation and enabled the sensor nodes to transmit the data directly to the sink without forwarding through the intermediate nodes. Those nodes are mostlythe cluster heads that transmits the data of its own cluster nodes and also forwards the data from the neighboring cluster heads. The relocation of the sink is periodic and the sensor nodes can predict the availability of sink in its region in the near future. The circular movement of the sink eliminates the multi hop transmission by the cluster heads. But the cluster heads suffer heavy energy loss due to the aggregation of data from the cluster nodes. The sensor nodes acting as cluster heads periodically changes, as a single node acting as cluster head for a longer duration tends to drain out its available energy. The selection of cluster head is an overhead as all the sensor nodes in a cluster are involved it the operation and consumes a significant amount of energy. When the sink moves in a linear path it eliminates the formation of the cluster and almost all the sensor nodes transmit the data directly to the sink through single hop transmission. Very few nodes that do not come under the coverage range of the sink transmit the data through the intermediate nodes in multi hop transmission. For future work the sink movement in linear path can be extended to multiple sink, the area covered by a single sink in a particular time would be minimum, whereas the use of multiple sink would increase the area covered and an effective data aggregation is possible. 


\section{References}

1. Ahmad, J., Khan, M. A., Hwang, S. O., \& Khan, J. S. (2017). A compression sensing and noise-tolerant image encryption scheme based on chaotic maps and orthogonal matrices. Neural Computing and Applications, 28, 953-967.

2. Al-Hayani, B., \& Ilhan, H. (2020a). Efficient cooperative image transmission in one-way multi-hop sensor network. International Journal of Electrical Engineering \& Education, 58, 1-17.

3. Al-Hayani, B., \& Ilhan, H. (2020b). Image transmission over decode and forward based cooperative wireless multimedia sensor networks for Rayleigh fading channels in medical internet of things (MIoT) for remote health-care and health communication monitoring. Journal of Medical Imaging and Health Informatics, 10, $160-168$.

4. Alshibani, D. R., \& Ibrahim, R. S. (2015). Implementation of gray image encryption using multilevel of permutation and substitution. International Journal of Applied Information Systems, 10(1), 25-30.

5. Chandrasekhar, N., Vasudevan, M., Bhaduri, A. K., \& Jayakumar, T. (2015). Intelligent modeling for estimating weld bead width and depth of penetration from infra-red thermal images of the weld pool. Journal of Intelligent Manufacturing, 26(1), 59-71

6. Ilhan, A., Karaköse, M., \& Akin, E. (2015). Combined intelligent methods based on wireless sensor networks for condition monitoring and fault diagnosis. Journal of Intelligent Manufacturing, 26(4), 717-729.

7. Krishna, P. R., Teja, C. V. M. S., Renuga Devi, S., \& Thanikaiselvan, V. (2018). A chaos based image encryption using Tinkerbell map functions. In Second international conference on electronics, communication and aerospace technology, Coimbatore (pp. 578-582).

8. Liu, M., Ma, J., Lin, L., Ge, M., Wang, Q., \& Liu, C. (2017). Intelligent assembly system for mechanical products and key technology based on internet of things. Journal of Intelligent Manufacturing, 28, 271-299.

9. Mahesh, C., Agarwal, D., \& Bansal, A. (2016). Image transmission through the wireless channel: A review. In IEEE International conference on power electronics, intelligent control and energy systems (ICPEICES).

10. Otazo, R., Candès, E., \& Sodickson, D. K. (2015). Low-rank plus sparse matrix decomposition for accelerated dynamic MRI with separation of background and dynamic components. Magnetic Resonance in Medicine, 73, 1125-1136.

11. Pappachan, J., \& Baby, J. (2015). Tinkerbell maps based image encryption using magic square. International Journal of Advanced Research in Electrical Electronis and Instrumentation Engineering, 4(7), 120-126.

12. Ponuma, R., \& Amutha, R. (2017). Compressive sensing based image compression-encryption using novel 1D-chaotic map. Multimedia Tools and Applications, 77, 19209-19234.

13. Singh, A. K., Dave, M., \& Mohan, A. (2016). Hybrid technique for robust and imperceptible multiple watermarking using medical images. Multimedia Tools and Applications, 75(14), 8381-8401.

14. Song, L., Alajaji, F., \& Linder, T. (2019). Capacity of burst noise-erasure channels with and without feedback and input cost. IEEE Transactions on Information Theory, 65, 276-291.

15. Stoyanov, B. (2016). Novel secure pseudo-random number generation scheme based on two Tinkerbell maps. Advanced Studies in Theoretical Physics, 9(9), 411-421.

16. Ur Rehman, Y. A., Tariq, M., \& Sato, T. (2016). A novel energy efficient object detection and image transmission approach for wireless multimedia sensor networks. IEEE Sensors Journal, 16(15), 5942-5949.

17. El-Bakary, E. M., Hassan, E. S., Zahran, O., El-Dolil, S. A., \& Abd El-Samie, F. E. (2013). Efficient image transmission with multi-carrier CDMA. Wireless Personal Communications, 69, 979-994.

18. Karakus, C., Gurbuz, A. C., \& Tavli, B. (2013). Analysis of energy efficiency of compressive sensing in wireless sensor networks. IEEE Sensors Journal, 13, 1999-2008.

19. Kwak, H. J., \& Park, G. T. (2014). Contrast enhancement for intelligent surveillance systems using multilocal histogram transformation. Journal of Intelligent Manufacturing, 25, 303-318.

20. Zhang, J., Zhao, D., Zhao, C., Xiong, R., Ma, S., \& Gao, W. (2012). Image compressive sensing recovery via collaborative sparsity. IEEE Journal on Emerging and Selected Topics in Circuits and Systems, 2, 380-391. 